\title{
Simple model relating recombination rates and non- proportional light yield in scintillators
}

\author{
G. Bizarri ${ }^{1}$, W.W. Moses $^{1}$, J. Singh $^{1 *}$, A.N. Vasil'ev ${ }^{2}$, and R.T. Williams ${ }^{3}$ \\ ${ }^{1}$ Lawrence Berkeley National Laboratory, Berkeley, CA 94720-8119, USA, and (Permanent address): School of Engi- \\ neering and IT, B-41, , Charles Darwin University, Darwin N.T. 0909, Australia.
}

${ }^{2}$ Institute of Nuclear Physics, Moscow State University, Moscow 119991, Russia

${ }^{3}$ Department of Physics, Wake Forest University, Winston-Salem, NC 27109, USA

Received zzz, revised zzz, accepted zzz

Published online zzz (Dates will be provided by the publisher.)

PACS 07.85.Fv, 28.52.Nh, 29.40.Mc, 29.40.-n

* Corresponding author: e-mail: jai.singh@cdu.edu.au, Phone: +61 889466 811, Fax: +61 889999999

\begin{abstract}
We present a phenomenological approach to derive an approximate expression for the local light yield along a track as a function of the rate constants of different kinetic orders of radiative and quenching processes for excitons and electron-hole pairs excited by an incident $\gamma$-ray in a scintillating crystal. For excitons, the radiative and quenching processes considered are linear and binary, and for electron-hole pairs a ternary (Auger type) quenching process is also taken into account. The local light yield $\left(Y_{L}\right)$ in photons per $\mathrm{MeV}$ is plotted as a function of the deposited energy, $-\mathrm{d} E / \mathrm{d} x(\mathrm{keV} / \mathrm{cm})$ at any point $x$ along the track length. This model formulation achieves a certain simplicity by using two coupled rate equations. We discuss the approximations that are involved. There are a sufficient number of parameters in this model to fit local light yield profiles needed for qualitative comparison with experiment.
\end{abstract}

1 Introduction The non-proportional light yield observed in scintillators has attracted research interest for decades [1] because of their applications in medical imaging [2], security scanners [3], radiation detectors [4], etc. The light yield $(Y)$ of a scintillator is defined as the total energy of photons emitted $\left(E_{p}\right)$ per unit energy deposited by an incident particle or a $\gamma$-ray [5]. The constancy of $\mathrm{Y}$ for a given material is operationally assumed for many scintillator applications, but in reality Y depends on the energy of the primary particle. The departure of $Y$ from a constant, versus deposited energy, is called the "nonproportionality" in the light yield of scintillators. Although scintillators have been known for many decades, the origin of non-proportionality is not yet clearly understood.

The initial interaction of, e.g., a $\gamma$ ray with a scintillator creates a hole and a very high energy excited electron. The electron then loses its energy in a cascade, creating excited electron-hole (e-h) pairs, typically at high concentration. Some of these excitations finally recombine radiatively and emit photons, i.e. the scintillator response. Creation of high density excitations in scintillators involves various types of radiative and non-radiative interaction processes among the excitations (in final stages these are, excitons, conduction or trapped electrons, and valence or trapped holes). Their ultimate probability of radiative recombination becomes a complicated function of the rates of all the interaction processes. As these rates vary in magnitude from one material to another, different materials can be expected to exhibit different forms of non-proportionality [6]. In order to understand the non-proportionality in scintillators, therefore, we need to know the rates of various processes of interaction occurring in a high excitation density situation in a scintillating crystal when it is subjected to high energy incident radiation.

In this paper, a phenomenological model is presented to study the scintillator response by including the rates of recombination processes that are $1^{\text {st }}$ and $2^{\text {nd }}$ order in exciton concentration and that are $1^{\text {st }}, 2^{\text {nd }}$, and $3^{\text {rd }}$ order in e-h pair creation. It is considered that initially one creates a high density of free e-h pairs and excitons. The interchange 
between e-h pairs and excitons in both directions is also possible and is considered.

A fundamental approximation in this model is that the recombination rate equations are considered to depend only on concentration of excitons and of electron-hole pairs. Assuming that the material remains neutral, all electrons and holes are created in pairs, including those that are excitons and those that can diffuse, be trapped, and ultimately recombine as independent carriers. A more precise model should also include the rate equations for independent electrons and holes. What we are trying to develop is a compact model yielding intuitive trends influenced by choices of a few identifiable physical parameters. We describe a model that attempts to achieve this, and examine the approximations involved. The model and solution of equations can be described only briefly in the length of this publication, so details will be published elsewhere [7].

2 Theory Electrons and holes must be excited pairwise in a material that remains neutral. We identify excitons as electron-hole pairs that undergo transport and trapping as a pair, and finally undergo first-order recombination if decaying radiatively. In this paper, we regard all other electrons and holes, capable of transporting independently and recombining independently, as belonging to the population of e-h pairs. Geminate recombination of e$\mathrm{h}$ pairs would be $1^{\text {st }}$ order in pair concentration, and it is perhaps semantic whether this differs from exciton decay. Recombination of e and $\mathrm{h}$ from independent pairs is clearly $2^{\text {nd }}$ order in pair concentration. The model proposed is a statement that the concentration of excitons and of e-h pairs at a point $x$ along the primary particle track, can be expressed by the following two rate equations:

$$
\begin{aligned}
& -\frac{d n_{e x}(x)}{d t}=\left(R_{1 x}+K_{1 x}\right) n_{e x}(x)+\left(R_{2 x}+K_{2 x}\right) n_{e x}^{2}(x) \\
& -\gamma_{e x} n_{e h}(x)+\gamma_{x e} n_{e x}(x)-f_{x} n(x) \delta(t),
\end{aligned}
$$

$$
\begin{aligned}
& -\frac{d n_{e h}(x)}{d t}=\left(R_{1 e h}+K_{1 e h}\right) n_{e h}(x)+\left(R_{2 e h}+\right. \\
& \left.K_{2 e h}\right) n_{e h}^{2}(x)-\gamma_{x e} n_{e x}(x)+\gamma_{e x} n_{e h}(x)+K_{3 e h} n_{e h}^{3}(x) \\
& -\left(1-f_{x}\right) n(x) \delta(t),
\end{aligned}
$$

where $n_{e x}(x)$ is the excitonic concentration and $n_{e h}(x)$ is the concentration of excited e-h pairs at any point $x$ on the beam track. $f_{x}$ is the fraction concentration of excitons, $\left(1-f_{x}\right)$ is the fraction concentration of e-h pairs, and $n(x) \delta(t)$ represents the total number of excitations, $n(x)=n_{e x}(x)+n_{e h}(x)$, created by the incident energy at time $t=0$ at any point $x$ along the track. . This concentra- tion is assumed to be unaffected by the branching in the track path and is given by $n(x)\left(\mathrm{cm}^{-3}\right)$ :

$$
n(x)=\frac{\left(-\frac{\partial E}{\partial x}\right)}{\pi r^{2} E_{e h}},
$$

where $E$ is the total initial energy incident at any point $x$, $\pi r^{2}$ is the average area of cross section of the track and $E_{e h}$ is the average energy required to create an excitation in a scintillator. Here, $\mathrm{E}_{\mathrm{eh}}$ is assumed to be three times the band gap energy: $E_{e h}=3 E_{g}[8] . R_{i x}$ and $K_{i x}$ denote the rates of radiative and non-radiative (quenching) recombinations, respectively, of excitons, and $i=1,2$, denote linear (1) and binary (2) rates. $R_{i e h}$ and $K_{i e h}(i=1,2)$ are the corresponding rates of radiative and quenching recombination for an e-h pair, and $K_{3 e h}$ is the rate of Auger-type (ternary) recombination of an e-h pair. The concept of an Auger process which is $3^{\text {rd }}$ order in e-h pair concentration follows the lines discussed earlier for geminate $\left(1^{\text {st }}\right.$ order $)$ and independent $\left(2^{\text {nd }}\right.$ order) recombination of e-h pairs. If the 3 particles - electron, hole, and electron - undergoing Auger decay all come from different initial e-h pairs, then it is $3^{\text {rd }}$ order in pair concentration. There can also be geminate Auger recombination, where two of the three particles are from the same e-h pair at creation. The kinetics of that are $2^{\text {nd }}$ order in pair concentration, but then this is simply another term contributing to the $\mathrm{K}_{2 \mathrm{eh}}$ quenching factor. $\gamma_{e x}$ and $\gamma_{x e}$ are the rates of converting an e-h pair into an exciton and vice versa, respectively. Depending on $f_{x}$, scintillators can be classified in three categories: (i) excitonic with $f(x)=1$, (ii) non-excitonic with $f(x)=0$, and (iii) mixed case $0<f(x)<1$.

If one integrates Eqs. (1) and (2) over time, there is no net change in $n_{e x}$ or $n_{e h}$ on the left from before the impact to long after it, and so one gets:

$$
\begin{aligned}
& 0=\left(R_{1 x}+K_{1 x}\right)<n_{e x}(x)>+\left(R_{2 x}+K_{2 x}\right)<n_{e x}^{2}(x)>(4) \\
& -\gamma_{e x}<n_{e h}(x)>+\gamma_{x e}<n_{e x}(x)>-f_{x} n(x) \\
& 0=\left(R_{1 e h}+K_{1 e h}\right)<n_{e h}(x)>+\left(R_{2 e h}+K_{2 e h}\right)<n_{e h}^{2}(x)> \\
& -\gamma_{x e}<n_{e x}(x)>+\gamma_{e x}<n_{e h}(x)>+K_{3 e h}<n_{e h}^{3}(x)>-\left(1-f_{x}\right) n(x)
\end{aligned}
$$

where $<n(x)>$ represents the integrated value of $n(x)$ over time. Adding Eqs. (4) and (5) we get:

$$
\begin{aligned}
& \left(R_{1 x}+K_{1 x}\right)<n_{e x}(x)>+\left(R_{2 x}+K_{2 x}\right)<n_{e x}^{2}(x)>+\left(R_{1 e h}+K_{1 e h}\right) \\
& \times<n_{e h}(x)>+\left(R_{2 e h}+K_{2 e h}\right)<n_{e h}^{2}(x)>+K_{3 e h}<n_{e h}^{3}>=n(x)
\end{aligned}
$$

Using Eq. (6), the local scintillator response or local light yield from a cross-section plane at any point $x$ along the track can be written as: 
$Y_{L}=\left(R_{1 x}<n_{e x}(x)>+R_{2 x}<n_{e x}^{2}(x)>+R_{1 e h}<n_{e h}(x)>\right.$

$\left.+R_{2 e h}<n_{e h}^{2}(x)>\right) / n(x)$

where the numerator consists of only terms of the radiative processes in Eq. (6). The yield in Eq. (7) has been defined previously as the local light yield at any given position $x$ along the track.

The quantities $<n_{e x}(x)>,<n_{e x}^{2}(x)>,<n_{e h}(x)>$ and $<n_{e h}^{2}(x)>$ are estimated here within the linear order approximation [7], that means by neglecting the binary and ternary terms in Eqs. (4) and (5). Accordingly the local light yield is obtained as:

$$
Y_{L}=\frac{a_{1}+a_{2} n(x)}{1+a_{3} n(x)+a_{4} n(x)^{2}},
$$

where

$$
\begin{gathered}
a_{1}=\frac{\left(R_{1 x} A+R_{1 e h} B\right)}{D} ; \quad D=\left(R_{1 x}+K_{1 x}\right) A+\left(R_{1 e h}+K_{1 e h}\right) B, \\
a_{2}=\frac{\left(R_{2 x} A^{2} / \tau_{x}+R_{2 e h} B^{2} / \tau_{e h}\right)}{2 D}, \\
a_{3}=\frac{\left(R_{2 x}+K_{2 x}\right) A^{2} / \tau_{x}+\left(\left(R_{2 e h}+K_{2 e h}\right) B^{2} / \tau_{e h}\right.}{2 D},(9 \mathrm{c}) \\
a_{4}=\frac{K_{3 e h} B^{3}}{3 D \tau_{e h}^{2}}, \quad(9 \mathrm{~d}) \\
1 / \tau_{e x}=R_{1 x}+K_{1 x} \text { and } 1 / \tau_{e h}=R_{1 e h}+K_{1 e h}, \quad(9 \mathrm{e}) \\
A=\frac{\left[\gamma_{e x}+\left(R_{1 e h}+K_{1 e h}\right) f_{x}\right]}{\left(R_{1 x}+K_{1 x}\right)\left(R_{1 e h}+K_{1 e h}\right)+\gamma_{e x}\left(R_{1 x}+K_{1 x}\right)+\gamma_{x e}\left(R_{1 e h}+K_{1 e h}\right)},(9 \mathrm{f}) \\
B=\frac{\left[\left(1-f_{x}\right)\left(R_{1 x}+K_{1 x}\right)+\gamma_{x e}\right]}{\left(R_{1 x}+K_{1 x}\right)\left(R_{1 e h}+K_{1 e h}\right)+\gamma_{e x}\left(R_{1 x}+K_{1 x}\right)+\gamma_{x e}\left(R_{1 e h}+K_{1 e h}\right)}
\end{gathered}
$$

Provided the rates are known, substituting Eqs. 9 (a-g) in Eq. (8) the local yield can be calculated as a function of $\mathrm{d} E / \mathrm{d} x(\mathrm{keV} / \mathrm{cm})$ through Eq. (3) for any scintillating crystal.

However, such a profile of local light yield does not correspond directly to experimental profiles of total light yield versus primary particle energy, as it is usually presented. Murray and Meyer [6] obtained an experimental curve which could be presented as an approximation to the profile of light yield vs $(-\mathrm{dE} / \mathrm{dx})$ by employing primary particles of widely different ionization rates ( $\gamma$-rays (electrons), protons, deuterons, $\alpha$ particles, and various heavy ions) and assigning a $\mathrm{dE} / \mathrm{dx}$ characterizing the stopping power of the scintillator for that particle and energy. More recent detailed results obtained with Compton scattered primary electrons really produce total light yield integrated over the whole track with associated energy deposition densities, plotted versus primary particle energy. Properly, one should integrate the model results for local light yield using a prescription for $\mathrm{dE} / \mathrm{dx}$ such as the Be-
the-Block equation [8]. Since the aim of this report is to try to describe qualitative trends with few parameters, results will be left in terms of local light yield.

Table.1 Rates $\left(\mathrm{s}^{-1}\right)$ of radiative and quenching processes used in the calculation of light yield in NaI scintillators at $300 \mathrm{~K}$.

\begin{tabular}{llll}
\hline$R_{1 x}$ & $K_{1 x}$ & $R_{1 e h}$ & $K_{1 e h}$ \\
$3 \times 10^{4}$ & 0 & 0 & $2.193 \times 10^{4}$ \\
\hline$R_{2 x}\left(\mathrm{~cm}^{3}\right)$ & $R_{2 e h}\left(\mathrm{~cm}^{3}\right)$ & $\gamma_{e x}$ & $\gamma_{x e}$ \\
0.0 & $1.85 \times 10^{-4}$ & $10^{3}$ & 98.3 \\
\hline$K_{2 x}\left(\mathrm{~cm}^{3}\right)$ & $K_{2 e h}\left(\mathrm{~cm}^{3}\right.$ & $K_{3 x}\left(\mathrm{~cm}^{6}\right.$ & $K_{3 e h}\left(\mathrm{~cm}^{6}\right)$ \\
& ) & ) & $1.69 \times 10^{-11}$ \\
$1.85 \times 10^{-4}$ & 0.0 & 0.0 & \\
\hline
\end{tabular}

3 Results and Discussion It may be taken as obvious that with 4 available parameters, Eq. (8) is capable of being fit to any of a wide variety of profiles plotting local yield $Y_{L}$ vs deposited energy density (proportional to $\mathrm{dE} / \mathrm{dx}$ ). Nevertheless for purposes of illustration we have plotted $\mathrm{Y}_{\mathrm{L}}$ for three cases: (i) pure excitonic, $f_{x}=1$, (ii) pure e-h pairs, $f_{x}=0$ and (iii) mixed case $f_{x}=0.5$. The rates used for calculating the yield are estimated from references [9 -14]. The curve with $f_{x}=0$ represents the local profile qualitatively similar to the light yield of $\mathrm{NaI}$ :Tl vs $\mathrm{dE} / \mathrm{dx}$ due to Meyer and Murray [6] and more recent results of SLYNCI experiments [12]. The important features reproduced in the model curve are the characteristic rising slope at small $\mathrm{dE} / \mathrm{dx}$ and the falling slope at large $\mathrm{dE} / \mathrm{dx}$. The latter feature is common to all known scintillators and appears in all

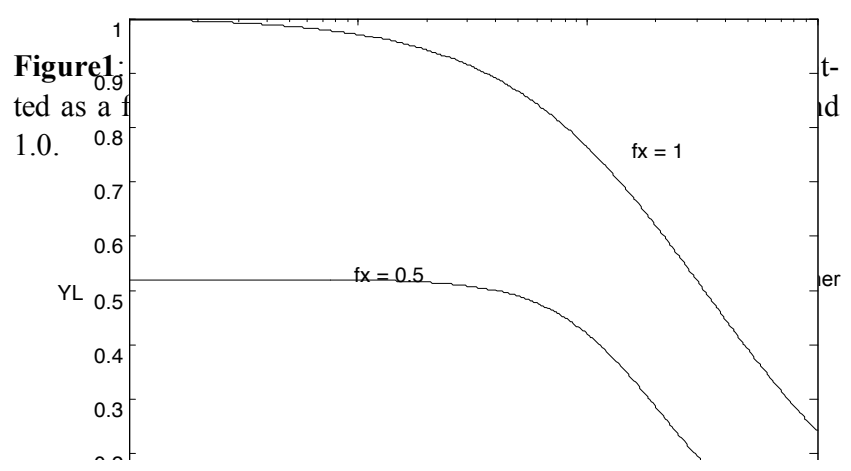


three cases shown in Fig. 1. The former case (rising slope at low $-\mathrm{dE} / \mathrm{dx}$ ) is peculiar to activated halide scintillators among those investigated so far as it appears with $f=0$. The other two curves with $f=0.5$ and 1.0 displayed in Fig. 1 illustrate the more general behavior for materials other than activated halides. The local yield is flat at low $\mathrm{dE} / \mathrm{dx}$ and finally rolls off at high $\mathrm{dE} / \mathrm{dx}$.

This simple model including only the concentrations $n_{e x}$ and $n_{e h}$ can, in the sense of a parameter fit, reproduce the important qualitative features and trends of local yield profiles in scintillators. The next two questions are whether the parameter values resulting from the fit can be related to physical characteristics of the materials, and if so whether the values correspond to measured values, or seem reasonable if there are no measurements yet. The real goal of this admittedly approximate model is whether its simplicity and minimal set of parameters can allow one to capture an intuitive picture of a trend that remains tantalizing: Why do some of the activated halide scintillators, particularly, show the rising slope at low $\mathrm{dE} / \mathrm{dx}$, while most other scintillators do not? Apparently scintillators with pure e-h pairs behaviour show a clear rising slope. This will be pursued further in Ref. [7].

Acknowledgements This work was supported by the National Nuclear Security Administration, Office of Defense Nuclear Nonproliferation, Office of Nuclear Nonproliferation Research and Engineering (NA-22) of the U.S. Department of Energy under Contract No. DE-AC02-05CH11231, grant number NNSA LB06-316-PD05 / NN2001000. We gratefully acknowledge the technical assistance from Ms Francesca Morlino in preparing the manuscript.

\section{References}

[1] W. W. Moses, S. A. Payne, W.-S. Choong, G. Hull, and B. W. Reutter, IEEE Trans. Nucl. Science (2007) in press.

[2] W. W. Moses, Proc. International Conference on Inorganic Scintillators and their Applications, SCINT'99, Eds. V. Mikhailim, (Moscow, Russia, 1999).

[3] E. R. Siciliano, J. H. Ely, R. T. Kouzes, B. D. Milbrath, J. E. Scheppe and D.C. Stromswold, Nucl. Instr. Methods in Physics Research A 550, 647 (2005).

[4] M. Globus, B. Grinyov and J. K. Kim, Inorganic Scintillators for Modern and Traditional Applications (Institute for Single Crystals, Kharkov, Ukraine, 2005).

[5] See, e.g., J.D. Valentine, B.D. Rooney and P. Dorenbos, IEEE Trans. Nucl. Science 45, 1750 (1998).

[6] R.B.Murray and A. Meyer, Phys. Rev. 112, 815 (1961).

[7] G. Bizarri, W.W. Moses, J. Singh, A.N. Vasil'ev, and R.T. William, (2008) to be published

[8] see, e.g., G. F. Knoll, Radiation Detection and Measurement, 3rd Ed. (John Eiley and Sons, N.Y., 2005) p. 31.

[9] D.J. Robbins, J. Electrochem. Soc.: Solid-State Sci. Tech. 127, 2694 (1980).

[10] F.S. Eby and W. K. Jenstschke, Phys. Rev. 96, 911 (1954).

[11] H. Nishimura, S. Nagata, J. Lumin. 40\& 41, 429 (1988)..

[12] G. Bizarri, N.J. Cherepy, W.S. Choong, G. Hull, W.W. Moses, S.A. Payne, J. Singh, J. D. Valentine, A.N. Vasil'ev and R.T. Williams, presented at SORMA'08, Berkeley, CA, USA.

[13] W.L. Emkey, A.B.Romberger, and W.J. Van Sciver, Phys.Rev. B20, 5326(1979).

[14] H.B. Dietrich, A.E. Purdy, R.B. Murray and R.T. Williams, Phys.Rev. B12, 5894 (1973). 